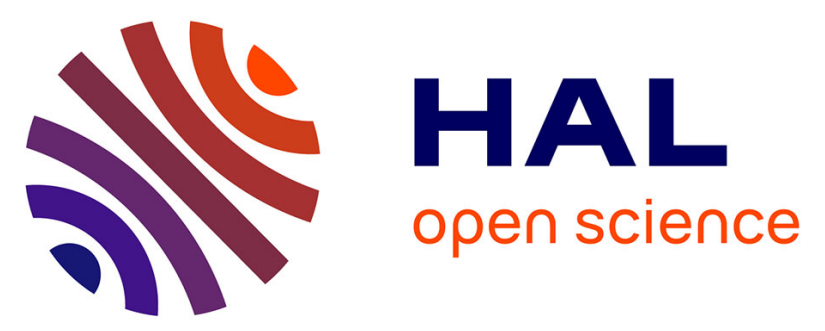

\title{
A new approach to sample entropy of multi-channel signals: application to EEG signals
}

Mohamad El Sayed Hussein Jomaa, Patrick van Bogaert, Nisrine Jrad, Marcelo Colominas, Anne Humeau-Heurtier

\section{- To cite this version:}

Mohamad El Sayed Hussein Jomaa, Patrick van Bogaert, Nisrine Jrad, Marcelo Colominas, Anne Humeau-Heurtier. A new approach to sample entropy of multi-channel signals: application to EEG signals. EUSIPCO 2018. 26th European Signal Processing Conference, Sep 2018, Rome, Italy. pp.1959-1963, 10.23919/EUSIPCO.2018.8553095 . hal-02159173

\section{HAL Id: hal-02159173 \\ https://hal.science/hal-02159173}

Submitted on 21 Jun 2021

HAL is a multi-disciplinary open access archive for the deposit and dissemination of scientific research documents, whether they are published or not. The documents may come from teaching and research institutions in France or abroad, or from public or private research centers.
L'archive ouverte pluridisciplinaire HAL, est destinée au dépôt et à la diffusion de documents scientifiques de niveau recherche, publiés ou non, émanant des établissements d'enseignement et de recherche français ou étrangers, des laboratoires publics ou privés. 


\title{
A New Approach to Sample Entropy of Multi-channel Signals: Application to EEG Signals
}

\author{
Mohamad El Sayed Hussein Jomaa, Patrick Van Bogaert, Nisrine Jrad, \\ Marcelo A. Colominas, and Anne Humeau-Heurtier \\ LARIS - Laboratoire Angevin de Recherche en Ingénierie des Systèmes, Université d'Angers, France \\ mohamad.elsayedhusseinjomaa@etud.univ-angers.fr
}

\begin{abstract}
In this paper, we propose a new algorithm to calculate sample entropy of multivariate data. Over the existing method, the one proposed here has the advantage of maintaining good results as the number of channels increases. The new and already-existing algorithms were applied on multivariate white Gaussian noise signals, pink noise signals, and mixtures of both. For high number of channels, the existing method failed to show that white noise is always the most irregular whereas the proposed method always had the entropy of white noise the highest. Application of both algorithms on MIX process signals also confirmed the ability of the proposed method to handle larger number of channels without risking erroneous results. We also applied the proposed algorithm on EEG data from epileptic patients before and after treatments. The results showed an increase in entropy values after treatment in the regions where the focus was localized. This goes in the same way as the medical point of view that indicated a better health state for these patients.
\end{abstract}

Index Terms—sample entropy, multivariate, complexity, EEG, epilepsy

\section{INTRODUCTION}

Studying signals of living systems is an important step towards better understanding the physiological and pathological functioning of these systems. This is particularly relevant when studying brain signals from electroencephalography (EEG). Many works describe the analysis of irregularity or complexity of EEG data (with multiscale and even multivariate approaches) [1] [2].

One of the common methods for studying irregularity of time series is entropy. Several algorithms have been proposed to study entropy, such as the approximate entropy [3], sample entropy [4], fuzzy entropy [5], and permutation entropy [6]. Sample entropy is one of the common entropy methods to be applied on EEG signals. A multivariate approach of sample entropy has also been proposed to consider the multivariate characteristics of EEG signals [7]. For a small number of channels or variates ( 2 or 3 ) this approach gives remarkable results. However as the number of channels increases, this approach fails to give results that could be depended on.

Therefore, to address the drawbacks of the existing multivariate approach, we propose in this paper a new algorithm to compute sample entropy for multivariate data. It shows

Thanks to the European Commission, Project FP7 DESIRE (Health-F2602531-2013) for supporting this work. M. El Sayed Hussein Jomaa and M. A. Colominas are both full-time granted by the DESIRE project. a higher ability of handling signals with high numbers of channels without risking the significance of the values.

This paper is organized as follows. We describe some of the existing sample entropy methods in Sec. II. We then describe the new proposed approach in Sec. III. This approach is validated on synthetic signals in Sec. IV. We present and discuss results obtained on EEG data in Sec.V. Finally Sec. VI concludes the paper.

\section{EXISTING SAMPLE ENTROPY METHOdS}

\section{A. Sample Entropy}

Sample entropy (SampEn) was first introduced by Richman and Moorman in 2000 [4]. It is the negative logarithm of the conditional probability that a time series of length $N$, showing similarity within a tolerance $r$ for length $m$, will also show similarity within a tolerance $r$ for length $m+1$, without considering self-matches. To calculate the SampEn of a time series $\left\{x_{1}, \ldots, x_{i}, \ldots, x_{N}\right\}$ of length $N$, first the delay vectors (DV) should be generated. For a given embedding dimension $m$ the DVs will be $\mathbf{X}_{m}(i)=\left[x_{i}, x_{i+1}, \ldots, x_{i+(m-1)}\right]$ where $i=1,2, \ldots, N-(m-1)$. For a given threshold or tolerance $r$, calculate all the possible distances between pairs of DVs $\left(\mathbf{X}_{m}(i)\right.$ and $\mathbf{X}_{m}(j)$ with $i \neq j$ ) and count the number of pairs that have a distance less than the tolerance $r$. Any type of distance could be used here, but the most commonly used is the Chebyshev distance. $B_{m}(r)$ will denote the frequency of occurrence. Then, extend the embedding dimension from $m$ to $m+1$ and generate the new DVs. For those new DVs, recalculate the distances between all possible pairs and count those with distances less than $r$ and denote their frequency of occurrence by $B_{m+1}(r)$. SampEn will then be SampEn $=-\ln \frac{B_{m+1}(r)}{B_{m}(r)}$. The lower the value of SampEn, the more regular the series is, thus more predictable it is.

\section{B. Multivariate Sample Entropy}

Multivariate Sample Entropy was introduced by Ahmed et al. [7] [8]. Two multivariate extensions of the SampEn were proposed, the naive method [8] and the full method [7], with the full method being commonly used since it outperforms the naive one [8].

Suppose $\left\{x_{1}^{k}, \ldots, x_{i}^{k}, \ldots, x_{N}^{k}\right\}$ is a multivariate time series where $1 \leq k \leq K$ is the index of the variate and $N$ is the length of the time series. As in Sec. II-A, the first step 

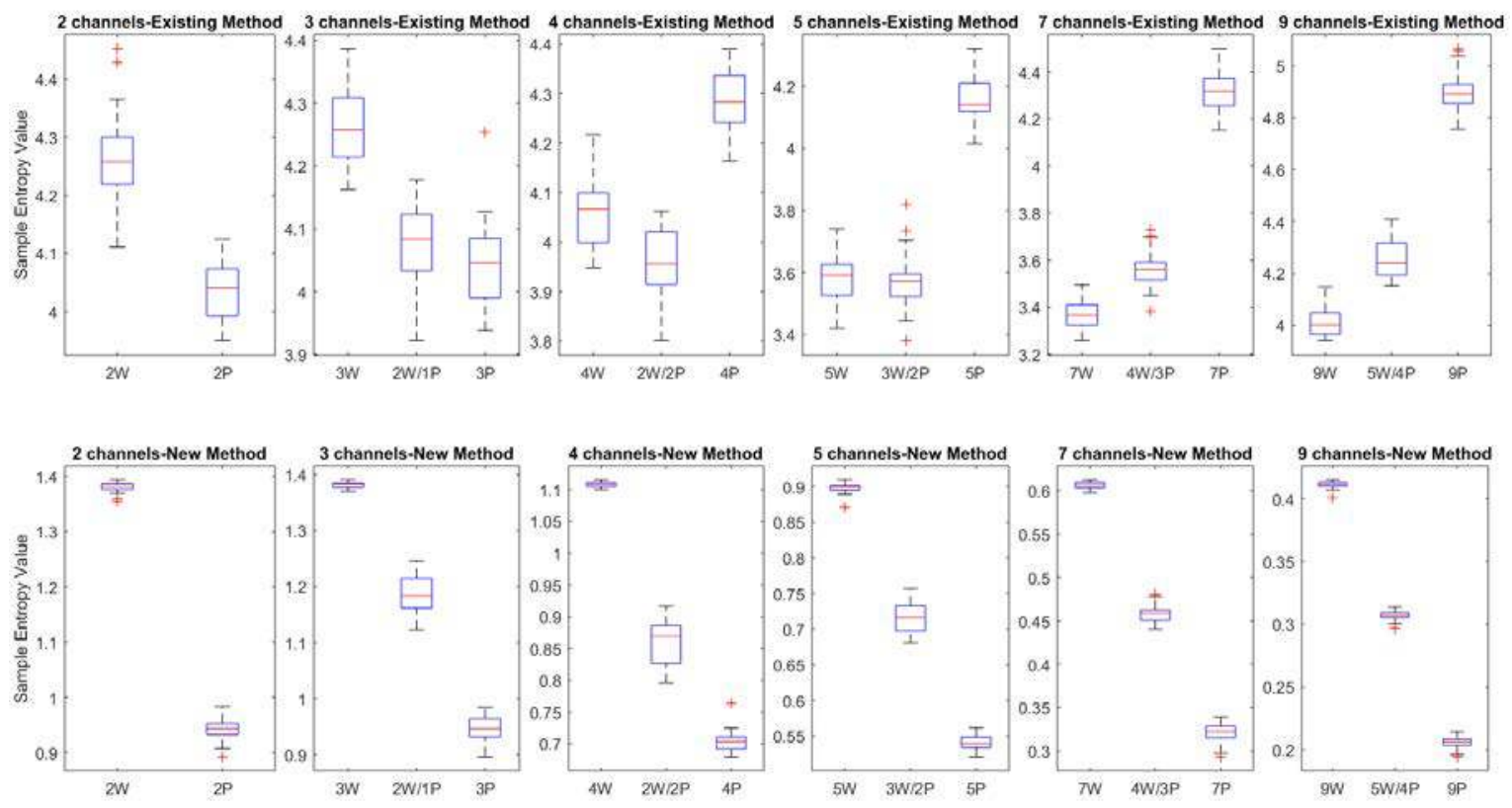

Fig. 1. Results of multivariate sample entropy for the two methods applied on white Gaussian (noted as W) and pink noise (noted as P), and a mixture of them (noted as $x \mathrm{~W} / y \mathrm{P}$ where $x$ is the number of white Gaussian noise channels and $y$ is the number of pink noise channels). Each signal had 5000 samples and 30 realizations were used. For both methods, the threshold was $r=0.15 \times$ standard deviation of the normalized signal and $m=2$ for all the channels.

to calculate the multivariate sample entropy is to generate the un-extended DVs: $\mathbf{X}_{\mathbf{M}}(i)=\left[x_{i}^{1}, \ldots, x_{i+\left(m_{1}-1\right)}^{1}, x_{i}^{2}, \ldots\right.$, $\left.x_{i+\left(m_{2}-1\right)}^{2}, \ldots, x_{i}^{K}, \ldots, x_{i+\left(m_{K}-1\right)}^{K}\right]$, where $i=1,2, \ldots, N-$ $\max \{\mathbf{M}\}$ and $\mathbf{M}=\left[m_{1}, m_{2}, \ldots, m_{K}\right]$ is the multivariate embedding dimension vector. Calculate the distances between all the possible pairs of DVs $\left(\mathbf{X}_{\mathbf{M}}(i)\right.$ and $\mathbf{X}_{\mathbf{M}}(j)$ with $\left.i \neq j\right)$ and count the number of distances that are less than a defined tolerance or threshold value $r$ and the frequency of occurrence of that would be denoted by $B_{\mathbf{M}}(r)$.

To extend the embedding dimension vector $\mathbf{M}$ to $\mathbf{M}+1$ in the full method $K$ DV subspaces are generated where in each subspace the embedding dimension of only one variate, $k$, is extended from $m_{k}$ to $m_{k}+1$. This way the extended DV subspace for $k$ would be $\mathbf{X}_{\mathbf{M}+1}^{k}(i)=$ $\left[x_{i}^{1}, \ldots, x_{i+\left(m_{1}-1\right)}^{1}, \ldots, x_{i}^{k}, \ldots, x_{i+\left(m_{k}-1\right)}^{k}, x_{i+\left(m_{k}\right)}^{k} \ldots, x_{i}^{K}\right.$ $, \ldots, x_{i+\left(m_{K}-1\right)}^{K}$. The pair-wise distances are calculated between all the DVs across all the $K$ subspaces and the number of pairs with distances less than the threshold $r$ is counted. The frequency of occurrence for that would be denoted by $B_{\mathbf{M}+1}(r)$. This way, the multivariate sample entropy is $m v S a m p E n=-\ln \frac{B_{\mathbf{M}+1}(r)}{B_{\mathbf{M}}(r)}$.

\section{NEW APPROACH}

It was noticed that the multivariate sample entropy method explained in Sec. II-B shows erroneous results when the number of channels increases (see Figs. 1 and 2). To address this issue, we propose a new way to calculate sample entropy for multivariate time series.

Let $\left\{x_{1}^{k}, \ldots, x_{i}^{k}, \ldots, x_{N}^{k}\right\}$ be a multivariate time series of length $N$ with $1 \leq k \leq K$ being the index of the variate and let $\mathbf{M}=\left[m_{1}, m_{2}, \ldots, m_{K}\right]$ be the multivariate embedding dimension vector. To calculate the sample entropy for this multivariate signal:

1) For each variate $k$, generate the DV subspace: $\mathbf{X}_{m_{k}}^{k}(i)=$ $\left[x_{i}^{k}, x_{i+1}^{k}, \ldots, x_{i+\left(m_{k}-1\right)}^{k}\right]$, where $i=1,2, \ldots, N-$ $\left(m_{k}-1\right)$ and $m_{k}$ is the corresponding embedding dimension for the variate $k$.

2) For each variate $k$, calculate the distances between all the possible vector pairs. Any type of distance between vectors could be used. Here we use the Chebyshev distance as it is commonly used in previous papers dealing with sample entropy [4] [7] [8]. Since there are $N-\left(m_{k}-1\right)$ vectors this means there are $\alpha=$ $\frac{\left(N-\left(m_{k}-1\right)\right)\left(N-m_{k}\right)}{2}$ possible distances without including self-distances.

3) Count the number of instances $A_{m_{k}}^{k}(r)$ where the distances are less than a predefined threshold $r$.

4) Define the frequency of occurrence as $B_{m_{k}}^{k}(r)=$ $\frac{A_{m_{k}}^{k}(r)}{\alpha}$.

5) Extend the dimension from $m_{k}$ to $m_{k}+1$ and repeat steps 1 to 4 . The new number of possible distances between pairs is $\beta=\frac{\left(N-m_{k}\right)\left(N-m_{k}-1\right)}{2}$. So the frequency of occurrence for the extended case will be: $B_{m_{k}+1}^{k}(r)=\frac{A_{m_{k}+1}^{k}(r)}{\beta}$, where $A_{m_{k}+1}^{k}(r)$ is the number of instances that the extended DV pairs have distances less than $r$.

6) Repeat all the above steps for all the variates of the time series $1 \leq k \leq K$. 

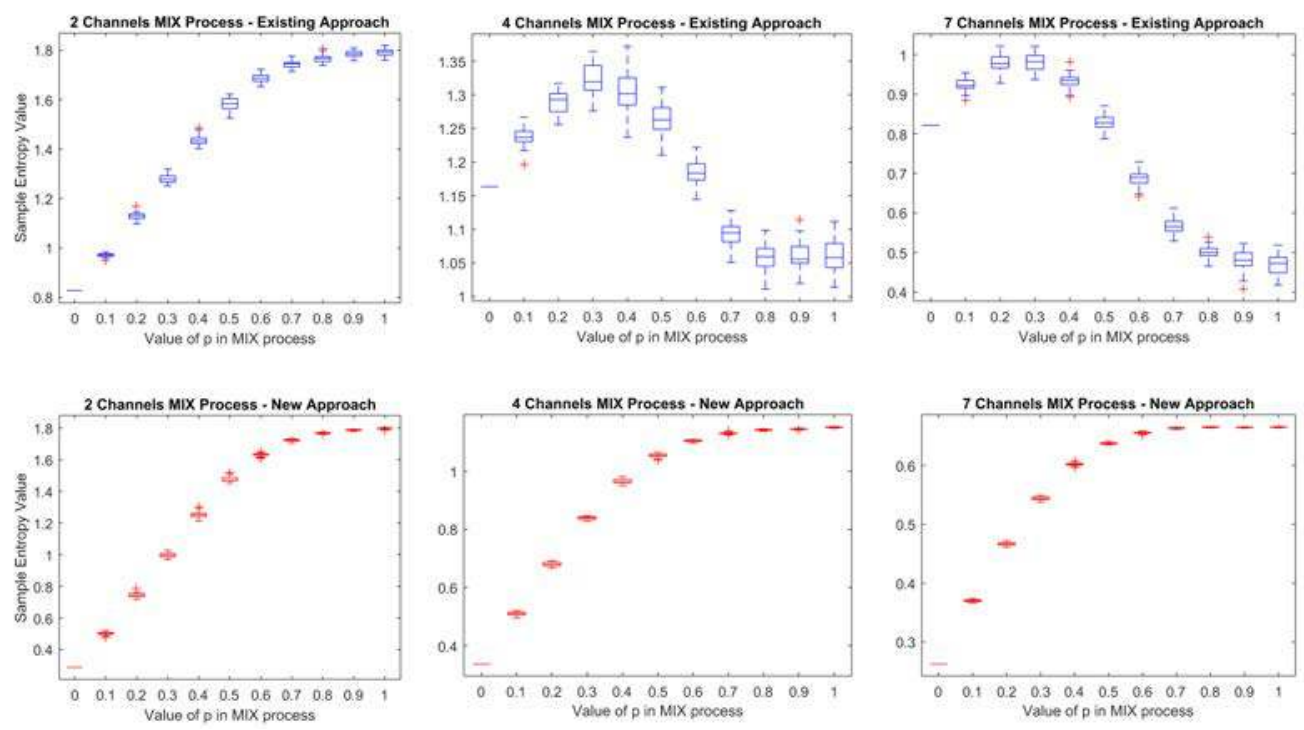

Fig. 2. Results of the two methods applied on MIX signals with varying number of channels. The value of $p$ ranging between 0 and 1 with a step 0.1 . Each signal had a length of 5000 samples and 30 realizations. For both methods, the threshold was $r=0.15 \times$ standard deviation of the normalized signal and $m=2$ for all the channels.

7) The sample entropy of the multivariate time series is:

$$
m v S a m p E n_{\text {new }}=-\ln \frac{\sum_{k=1}^{K} B_{m_{k}+1}^{k}(r)}{\sum_{k=1}^{K} B_{m_{k}}^{k}(r)} .
$$

The channel-wise computation of distances and frequencies of occurrence, followed by a global integration, makes this approach much more robust, as it will be confirmed immediately.

\section{VAlidation ON Synthetic Data}

To study the effect of increasing the number of channels on the existing multivariate sample entropy and the one we proposed, we apply both algorithms on synthetic data with varying number of channels and study the behavior of the results. The MATLAB code for the existing multivariate sample entropy was downloaded from [9].

\section{A. White and Pink Noise}

For this test, 3 types of multivariate noise signals are used:

1) Pure white Gaussian noise (WGN).

2) Pure pink noise (power spectrum proportional to $1 / f$ ).

3) A mixture of WGN and pink noise.

These signals have a length of $N=5000$ samples and each type has 30 realizations. The existing method and the new one were applied on these signals while the number of channels increased gradually from 2 to 9 . For both methods, the threshold was $r=0.15 \times$ standard deviation of the normalized signal and $M=\left[m_{k}\right]=2$ for $1 \leq k \leq K$.

Figure 1 shows that, for the existing method, the entropy value of the pure WGN signals is higher than that of the pure pink noise for signals with 2 and 3 channels. This is the normal case, since white noise is more irregular than pink noise [10]. But as the number of channels increases more than 3, the sample entropy value of white noise drops below that of the pink noise and the difference between white and pink noise becomes larger. Whereas for the new proposed method the sample entropy value of WGN remains larger than that of pink noise regardless of the number of channels of the noise signals. Also, the value of sample entropy for the mixtures of the two noises is always between those of pure WGN and pink noise. This proves that the new proposed method gives more consistent relative results and is not dependent on the number channels as the existing method is.

\section{B. MIX process}

In this test we evaluate the results of both methods as the multivariate signals gradually change from periodic to uniform random signals with respect to number of channels.

MIX process signals [3] are signals that range from periodic to completely random as the value of the parameter $p$ ranges from 0 to 1 . The two methods were applied on multivariate MIX processes with $0 \leq p \leq 1$ for various number of channels. The length of the signals was $N=5000$ samples and we used 30 realizations. For both methods, the threshold was $r=0.15 \times$ standard deviation of the normalized signal and $m=2$ for all the channels.

Figure 2 shows the results of the two methods on multivariate MIX process signals with 2, 4, and 7 channels. It is expected that sample entropy value is minimum for $p=0$ as the signals are purely periodical. This value should increase as the value of $p$ increases and the signal becomes more random. This is the case for both methods when the number of channels is 2. But as the number of channels increases the results of the existing method shows an increase in sample entropy value for $0 \leq p \leq 0.3$ but then the value starts decreasing for $0.3 \leq p \leq 1$ and even drops below the value of $p=0$. Whereas for the proposed method the value of sample entropy, as expected, increases as the value of $p$ increases. This, once 


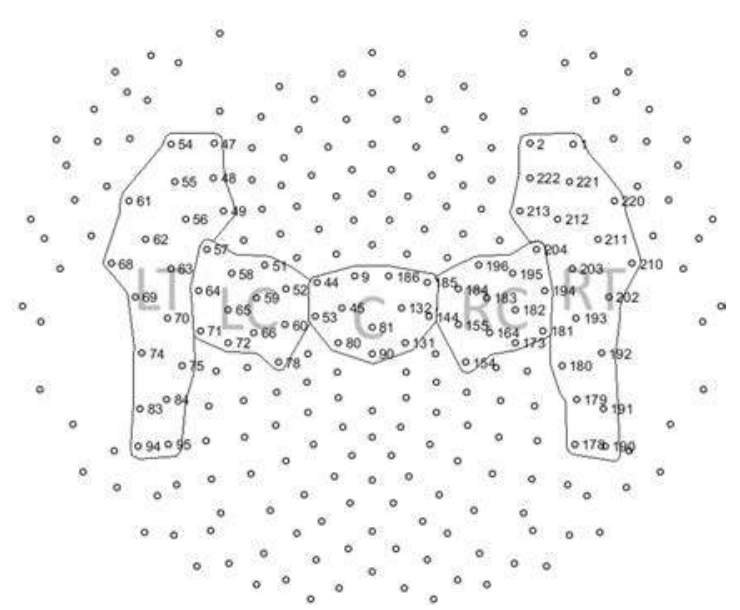

Fig. 3. Five regions where the entropy was calculated. LT: Left Temporal. LC: Left Central. C: Central. RC: Right Central. RT: Right Temporal.

again, shows that the proposed method is more consistent and is not dependent on the number of channels. It could therefore be applied for higher number of channels without risking getting erroneous values.

\section{Results on EEG DATA}

Epilepsy is a very commonly studied pathology using EEG. It is a neurological disorder characterized by epileptic seizures [11]. Besides, most epileptic patients present, in the interictal state, or the state when no seizures occur, interictal epileptiform discharges (IED). One of the common types of epilepsy in children is the benign childhood epilepsy with centrotemporal spikes (BECTS) [12]. It is known that children with such epilepsy recover as they grow older (around 14 to 18 years old) [13]. A less common epilepsy syndrome of childhood is epileptic encephalopathy with continuous spikes and waves during sleep (CSWS), that associates with cognitive impairment and strong IED during sleep [14]. Those two syndromes are quite similar.

When treating patients with those syndromes, clinicians aim to eliminate the cognitive deficits that are believed to be strongly related to the frequency of spikes [15]. Some antiepilepticdrugs (AED) use to lower the frequency of the spikes. For patients with BECTS a decrease in the number of spikes was reported when the patients were treated with sulthiame [16]. While the same effect took place on patients diagnosed with CSWS when they were treated with levetiracetam [17].

In this application we will focus on the resting state, without IED, EEG recordings of patients with the aforementioned syndromes. We will evaluate the sample entropy of the recorded EEG before and after treatment to see if it would imply an increase in irregularity after treatment, as it is known that signals from healthy subjects present higher irregularity than those of pathological subjects [18] [19].

\section{A. Recordings}

The EEG data of 2 epileptic patients recorded in Université Libre de Bruxelles; Hôpital Erasme (agreement of local ethical committee P2015/242) were analyzed. Patient 1 (10 years old male) was diagnosed with epilepsy with CSWS. The epileptic focus was clinically localized on T5 (10-20 system). Patient 2 (9 years old male) was diagnosed with BECTS with the focus being on C3 (10-20 system).

For each patient, two sessions of recordings were made, T0 being the baseline and T1 being 4 to 6 weeks after T0 while a certain AED was successfully administered in this period. Patient 1 was treated with lamotrigine, ethosuximide, and clobazam, while Patient 2 was treated with levetiracetam.

Both patients had their resting state EEG recorded while they were lying down for around 20 minutes. The acquisition was done using high density EEG (HD-EEG) with 256 electrodes (Electrical Geodesics, Inc.). The reference was the $\mathrm{Cz}$ electrode and the sampling frequency was $1000 \mathrm{~Hz}$.

\section{B. Preprocessing and Application}

All the recordings were preprocessed in the same way. A high order band-pass filter between $0.5 \mathrm{~Hz}$ and $45 \mathrm{~Hz}$ with a transition bandwidth of $0.22 \mathrm{~Hz}$ was applied. Then, independent component analysis (ICA) [20] was performed in order to isolate artifact-related components and discard them before reconstructing the artifact-free signals. Finally, the signals were visually examined by a trained neurophysiologist to identify the spikes, and 40 spike-free epochs with eyes open (1 second long) were extracted from each session recording.

Five regions of interest were taken from the 256 electrodes (Central (C), Left Central (LC), Right Central (RC), Left Temporal (LT), and Right Temporal (RT)). Figure 3 shows the position of those regions with respect to the whole net. We are interested in those regions because this is where we expect the irregularity change to take place since the foci, as mentioned in Sec. V-A, are central and temporal (since it is the nature of the syndromes). The new proposed sample entropy method was applied on the epoched multivariate signals of those five regions. The threshold was $r=0.15 \times$ standard deviation of the normalized signal and $m=2$ for all the channels.

\section{Results and Discussion}

For both patients, the sample entropy results are presented in Fig. 4. To objectively define the significance of the entropy changes, a Friedman test was applied on the results. Those regions with $p$-values $<0.02$, with Bonferroni correction for multiple comparisons, are considered to have significant differences between $\mathrm{T} 0$ and $\mathrm{T} 1$, and are marked with '*'.

For Patient 1, C, LC, and LT regions show a significant increase in sample entropy value. This suggests that the patient is responding with the medication and is approaching the healthy case [18] [19]. Besides, the regions that showed the significant increase are in correspondence with the clinical localization of the focus (T5). Patient 2 shows significant increase for only the LC region. This could have the same explanation as of Patient 1. The region of significance is also in correspondence with the clinical localization of the focus (C3). These results confirm the findings done in a previous study [21] as the irregularity of the EEG signals increased after almost one month of treatment. 

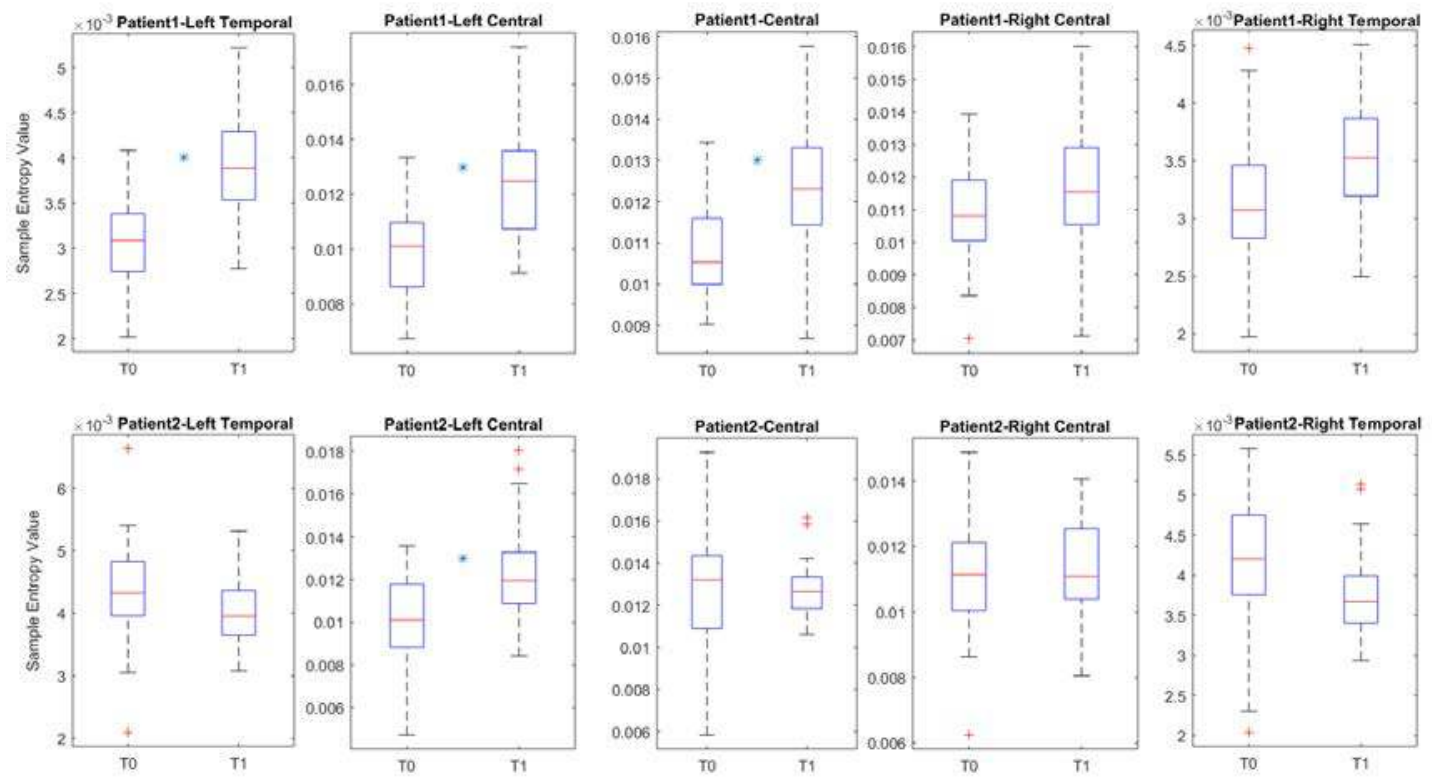

Fig. 4. Results obtained with the proposed sample entropy method on 40 epochs of two sessions for two subjects of the 5 regions. Epochs are 1 second long $(1000 \mathrm{~Hz})$. The threshold was $r=0.15 \times$ standard deviation of the normalized signal and $m=2$ for all the channels. Regions with statistically significant differences between T0 and T1 are marked with '*'. Friedman test was applied with significance threshold $p$-value $=0.02$ with Bonferroni correction.

\section{CONCLUSION}

Sample entropy has been commonly used in several fields, especially medical. In this paper, we reviewed the sample entropy approach and its existing multivariate extension and proposed a new approach for sample entropy of multivariate signals. Existing and proposed methods were applied on synthetic data. Results showed that the proposed method overcomes the drawback of the existing method that it fails to handle relatively large numbers of channels. Moreover, proposed method was applied to real EEG data verifying reliability of the method.

For future work, this method could be adopted for other entropy measures to be extended to the multivariate approach.

\section{REFERENCES}

[1] H. Adeli, S. Ghosh-Dastidar, and N. Dadmehr, "A wavelet-chaos methodology for analysis of EEGs and EEG subbands to detect seizure and epilepsy," IEEE Trans. Biomed. Eng., vol. 54, no. 2, pp. 205-211, 2007.

[2] A. Humeau-Heurtier, "The multiscale entropy algorithm and its variants: A review," Entropy, vol. 17, no. 5, pp. 3110-3123, 2015.

[3] S. M. Pincus, "Approximate entropy as a measure of system complexity.," Proc. Natl. Acad. Sci. USA, vol. 88, no. 6, pp. 2297-2301, 1991.

[4] J. S. Richman and J. R. Moorman, "Physiological time-series analysis using approximate entropy and sample entropy," Am. J. Physiol-Heart C., vol. 278, no. 6, pp. H2039-H2049, 2000.

[5] W. Chen, Z. Wang, H. Xie, and W. Yu, "Characterization of surface emg signal based on fuzzy entropy," IEEE Trans. Neur. Sys. Reh., vol. 15, no. 2, pp. 266-272, 2007.

[6] C. Bandt and B. Pompe, "Permutation entropy: a natural complexity measure for time series," Phys. Rev. Lett., vol. 88, no. 17, p. 174102, 2002.

[7] M. U. Ahmed and D. P. Mandic, "Multivariate multiscale entropy: A tool for complexity analysis of multichannel data," Phys. Rev. E, vol. 84, no. 6 , p. $061918,2011$.
[8] M. U. Ahmed, L. Li, J. Cao, and D. P. Mandic, "Multivariate multiscale entropy for brain consciousness analysis," in 2011 An. Int. Conf. IEEE EMBS (EMBC 2011), pp. 810-813, IEEE, 2011.

[9] "Dynamical complexity of multichannel data, multivariate multiscale entropy (mmse), matlab and data." http://www.commsp.ee.ic.ac.uk/ $\sim$ mandic/research/Complexity_Stuff.htm. 2011.

[10] M. Costa, A. L. Goldberger, and C.-K. Peng, "Multiscale entropy analysis of complex physiologic time series," Phys. Rev. Lett., vol. 89 , no. 6, p. 068102, 2002.

[11] R. S. Fisher et al., "ILAE official report: a practical clinical definition of epilepsy," Epilepsia, vol. 55, no. 4, pp. 475-482, 2014.

[12] U. Kramer, "Atypical presentations of benign childhood epilepsy with centrotemporal spikes: a review," J. Child Neurol., vol. 23, no. 7, pp. 785-790, 2008.

[13] L. M. Chahine and M. A. Mikati, "Benign pediatric localization-related epilepsies," Epileptic Disord., vol. 8, no. 3, pp. 169-183, 2006.

[14] P. Van Bogaert, "Epileptic encephalopathy with continuous spike-waves during slow-wave sleep including Landau-Kleffner syndrome," in Handbook of clinical neurology, vol. 111, pp. 635-640, Elsevier, 2013.

[15] J. Weglage, A. Demsky, M. Pietsch, and G. Kurlemann, "Neuropsychological, intellectual, and behavioral findings in patients with centrotemporal spikes with and without seizures," Dev. Med. Child Neurol., vol. 39, no. 10, pp. 646-651, 1997.

[16] T. Bast et al., "The influence of sulthiame on EEG in children with benign childhood epilepsy with centrotemporal spikes (BECTS)," Epilepsia, vol. 44, no. 2, pp. 215-220, 2003.

[17] A. Aeby, N. Poznanski, D. Verheulpen, C. Wetzburger, and P. Van Bogaert, "Levetiracetam efficacy in epileptic syndromes with continuous spikes and waves during slow sleep: experience in 12 cases," Epilepsia, vol. 46, no. 12, pp. 1937-1942, 2005.

[18] X.-S. Zhang, R. J. Roy, and E. W. Jensen, "EEG complexity as a measure of depth of anesthesia for patients," IEEE Trans. Biomed. Eng., vol. 48, no. 12, pp. 1424-1433, 2001.

[19] W. Bosl, A. Tierney, H. Tager-Flusberg, and C. Nelson, "EEG complexity as a biomarker for autism spectrum disorder risk," BMC Med., vol. 9, no. 1, p. 18, 2011.

[20] A. Hyvärinen, J. Karhunen, and E. Oja, Independent component analysis, vol. 46. John Wiley \& Sons, 2004.

[21] M. A. Colominas, M. E. S. H. Jomaa, N. Jrad, A. Humeau-Heurtier, and P. Van Bogaert, "Time-varying time-frequency complexity measures for epileptic EEG data analysis," IEEE Trans. Biomed. Eng., vol. Early Access, 2017. 